\title{
VALORES EXPRESADOS EN ESTUDIANTES DE ENFERMERÍA. TEMUCO - CHILE, 2009 ${ }^{1}$
}

\author{
VALUES EXPRESSED IN STUDENTS OF NURSING. \\ TEMUCO - CHILE, 2009
}

\author{
EDITH Rivas R.* \\ ANGÉlica Rivas L. ${ }^{* *}$ \\ Luis Bustos M. ${ }^{* *}$
}

\begin{abstract}
RESUMEN
Introducción: Los valores y las competencias en las(os) enfermeras(os) son facilitadores de la socialización profesional y elementales en la toma de decisiones. Objetivos: Relacionar los valores personales expresados en alumnos de cuarto semestre de la Carrera de Enfermería de la Universidad de La Frontera (UFRO) el 2009 con sus aspectos sociodemográficos y curriculares y con el perfil educacional-laboral de sus padres. Metodología: Estudio transversal-correlacional, muestra intencionada de 70 alumnos de cuarto semestre de Enfermería. Recopilación de información a través del instrumento Perfil de Valores Personales (Portrait Values Questionnaire). Resultados: En Escala de Valores Centrales, la puntuación promedio total fue de 4,8, considerada importante, según escala Likert, con un rango de 3,6 a 6,7. En Escala de Valores Secundarios, la puntuación promedio total fue de 5, considerada importante, con un rango de 3,6 a 6,7. Relación significativa entre Valores Centrales y nivel de avance curricular $(p=0,026)$ y entre etnia mapuche con Valores Secundarios $(p<0,0001)$. Conclusiones: Los valores centrales fueron considerados importantes por los alumnos estudiados, donde el valor dar sentido a la vida presenta la mayor puntuación promedio. Además, se observó diferencia significativa entre estos valores y el haber repetido cursos previamente. Asimismo, en valores secundarios existió diferencia significativa entre la etnia mapuche y estos valores. El desarrollo de la persona se ve acrecentado por sus valores personales y profesionales, así la disciplina debe estar estructurada de tal manera que desde la perspectiva filosófica, del lenguaje y de valores se puedan generar estrategias activas y reflexivas que permitan orientar y situar al futuro profesional en armonía con su contexto social.
\end{abstract}

Palabras clave: Disciplina, valores sociales, enfermería.

\begin{abstract}
Introduction: The values and skills of nurses (I) are facilitators of professional socialization and basic for decision-making. Objectives: To relate personal values expressed in nursing students in their 4th semester, Universidad de La Frontera (UFRO). Temuco - Chile, 2009. Methodology: Cross-sectional, correlational study. Purposive sample of 70 students of fourth semester of nursing. Data collection, through the Personal Values Profile instrument (Portrait Values Questionnaire). Results: On a scale of core values, the total average score was 4.8, considered important, according to Likert scale, ranging from 3.6 to 6.7. Scale of secondary values, whose total average score was 5, considered important, with a range of 3.6 to 6.7. Significant Core Values relationship between level of advancement and curriculum $(\mathrm{p}=0.026)$ and between Mapuche with secondary values ( $\mathrm{p}<0.0001$ ). Conclusions: The Core Values were considered important by the students studied, where the value "giving meaning to life" has the highest average score. In addition, significant difference between these

\footnotetext{
${ }^{1}$ Proyecto de Investigación No DI08-0069. Financiado por la Dirección de Investigación de la Universidad de La Frontera. * Enfermera, Doctora en Enfermería, Universidad de La Frontera, Temuco. Departamento de Pediatría Cirugía Infantil, Facultad de Medicina, Universidad de La Frontera. Temuco, Chile. E-mail: erivas@ufro.cl

${ }^{* *}$ Enfermera, Magíster en Enfermería. Hospital Hernán Henríquez A. Temuco. E-mail: ayrivasl@gmail.com

*** Bioestadístico, Universidad de La Frontera, Temuco. E-mail: lbustos@ufro.cl
} 
values and have repeated courses previously, was observed. Also, as for secondary values, significant difference between the Mapuche and these values, was found. The personal development is enhanced by their personal and professional values and discipline must be structured so that from the philosophical perspective, language and values, can create strategies that enable active and reflective guide and put forward professional in harmony with its social context.

Key words: Discipline, social values, nursing

Fecha recepción: 24.05.10 Fecha aceptación: 22.07.11

\section{INTRODUCCIÓN}

Dentro de la Filosofía, los valores han sido estudiados desde las virtudes, considerando a la ética y la moral, consistiendo en la aplicación efectiva de las preferencias de valores al comportamiento humano concreto (1). En el ámbito de la Psicología (2) se plantea un sistema de necesidades, encontrándose en la cúspide las de realización personal, en esta jerarquía los valores actúan como organizadores y son guías de las conductas que los sujetos realizan para lograr satisfacer dichas necesidades. Otro autor sostiene que sirven como guías del comportamiento humano y se adquieren en procesos de socialización primaria (familia y grupo de pares) y secundaria (escuela y otras instituciones). Y definen la forma de interpretar las actitudes de los demás y nos dicen qué está bien y qué está mal, siendo útiles a nivel individual, ya que aportan una importante información sobre los comportamientos, actitudes e identidades de las personas (3).

En salud se requieren profesionales que manifiesten un claro saber-hacer, lo cual demanda una determinada responsabilidad y capacidad para dar respuesta a las necesidades en salud, beneficiando de manera directa a la sociedad $(4,5)$. De esta forma en las ciencias de la salud existe necesidad social de disponer de profesionales con preparación tanto desde la perspectiva científica y técnica como desde los fundamentos de la ética, exigencia a la que se debe dar respuesta desde el área de pregrado como de postgrado (6).

Dentro de este contexto, surgen los valores y competencias que la persona debe desarrollar, entendiéndose como fundamento central en la humanización de los cuidados. Weis y Schank (7) los mencionan como aspectos básicos para enfermeras(os) como facilitadores de la socialización profesional y elementales en la toma de decisiones. Según Scheler: "Los valores son cualidades independientes de los bienes: los bienes son cosas valiosas [...]. La independencia de los valores implica su inmutabilidad; los valores no cambian [...] son absolutos [...]. Sólo nuestro conocimiento de los valores es relativo; no los valores mismos" (8).

Actualmente, se visualiza una crisis de valores, fenómeno denominado "desorientación vital", en la cual las aspiraciones y creencias son concebidas con marcada individualidad en función del entorno (9). En esta línea, se han generado diversos dilemas que perjudican los valores culturales e influyen de manera negativa en la expresión de principios éticos, ocasionando pérdida de las prácticas de valores fundamentales, tales como la libertad, la igualdad y la justicia (10).

En relación a lo anterior, es preciso actuar con fidelidad creativa y coherente, expresado en ideas, palabras, promesas e ideales. "Cuando esto se logra, se superan los conflictos entre las diversas energías que alberga nuestro ser: las instintivas y las espirituales" (11). Dicho fenómeno, exige elaboración y demanda el cumplimiento de condiciones como: creación de 
modos de acercamiento a valores fundamentales; cultivo de la sensibilidad; disminución de la indiferencia y ceguera, y sentido de responsabilidad frente a la praxis (12).

\section{VALORES Y DISCIPLINA}

La enfermera(o) desempeña su rol en la gestión del cuidado en forma holística a través del ciclo vital, manifestando una actitud ética y humanista, valorando la discreción, responsabilidad consigo mismo y con quienes se relaciona profesionalmente, respetando características individuales y colectivas al proporcionar cuidados, valores y creencias en el trabajo con grupos multiculturales. Al respecto, autores (13) proponen que los valores son conceptos o creencias correspondientes a intenciones o comportamientos que trascienden las situaciones concretas, y sirven de guía para la selección o evaluación de comportamientos y acontecimientos priorizados en función de su importancia relativa (13).

De esta forma, surge la idea de plantear la pregunta de investigación: ¿Cuáles son los valores personales expresados por los alumnos de enfermería de cuarto semestre de la Carrera de Enfermería, Universidad de La Frontera (UFRO). Temuco - Chile, 2009?, con el propósito de plantear nuevas hipótesis para constituir bases para futuras líneas investigativas y contribuir en la generación de conocimiento enfermero, dada la relevancia de la temática en el mundo de hoy.

En virtud de la correspondencia entre los comportamientos o valores de los alumnos y su formación personal (rol de responsabilidad de los padres), se incluye el perfil educacional y laboral de sus padres.

De forma análoga se describe el perfil biodemográfico y aspectos curriculares de los alumnos, ya que los perfiles valóricos de las personas son importantes predictores de comportamientos, intereses y actitudes.

\section{Objetivo general}

- Relacionar los valores personales expresados en alumnos de cuarto semestre de la Carrera de Enfermería con sus aspectos sociodemográficos y curriculares y con el perfil educacional - laboral de sus padres. Universidad de La Frontera (UFRO). Temuco - Chile, 2009.

\section{Objetivos específicos}

- Caracterizar el perfil sociodemográfico de la muestra.

- Identificar el perfil educacional y laboral de los padres de los alumnos de la carrera de Enfermería de la Universidad de La Frontera

- Identificar valores centrales y secundarios expresados en alumnos de cuarto semestre de la Carrera de Enfermería de la Universidad de La Frontera

- Relacionar los valores centrales y secundarios, en relación a aspectos sociodemográficos y curriculares.

\section{MATERIAL Y MÉTODO}

Estudio de corte transversal, correlacional, en una muestra intencionada de 70 alumnos de cuarto semestre de la Carrera de Enfermería. La variable dependiente fueron los valores centrales y secundarios.

La recopilación de los datos fue realizada por dos investigadoras, en una sala previamente acondicionada, previa firma del consentimiento de los participantes, a través del instrumento Perfil de Valores Personales, (Portrait Values Questionnaire) (13). Instrumento que consta de 2 dimensiones, distribuidas en dos listas de 32 valores centrales y 27 secundarios, medidos en escala de -1 a 7 , en orden de importancia, donde el -1: opuesto a mis valores y 7 : de suma importancia. 
El análisis se efectuó en programa computacional STATA v11.0. Se realizó un primer nivel de análisis descriptivo: distribución de frecuencias, medias, promedios y desviación estándar y un segundo nivel de análisis inferencial: ttest para varianzas iguales y distintas. Nivel de significación de 5\%. El estudio es antesala de un estudio longitudinal y cumple con los principios éticos y de consentimiento informado.

\section{RESULTADOS}

Los resultados se expresan en un primer nivel desde el perfil sociodemográfico de los 70 alumnos solteros, de cuarto semestre de Enfermería, sin estudios previos al ingreso de la carrera, registrándose además el nivel educa- cional y actividad laboral de sus padres.

Así se tiene que el 20\% de los alumnos son hombres, siendo el promedio de edad 20,1 años, con una desviación estándar (DS) de 1,14 años y mediana de 20 años.

Respecto de la procedencia, un 91,2\% señaló origen urbano, provenientes en un $51,4 \%$ de zonas costeras, cordillera y del sur del país. El 17,1\% correspondió a la etnia mapuche.

En creencias, el 77,1\% manifestó profesar religión católica, evangélica o protestante y un 22,9\% no profesa religión. El 85,7\% no realizaba actividad deportiva.

Sólo el 48,6\% de los alumnos señaló vivir con ambos padres al momento de la encuesta. Un 14,3\% presentó repetición de asignaturas (Tabla 1).

En las madres, el 22,9\% presentó educación básica completa y el 17,1\% enseñanza

Tabla 1. Perfil sociodemográfico alumnos de Enfermería. Universidad de La Frontera, año 2009.

\begin{tabular}{lcc}
\hline Variables & f & $\%$ \\
\hline Mujeres & 56 & 80,0 \\
Procedencia urbana & 62 & 91,2 \\
Etnia no mapuche & 58 & 82,9 \\
Religión que profesa & & \\
Católica & 40 & 57,1 \\
Evangélica & 12 & 17,1 \\
Protestante & 2 & 2,9 \\
Ninguna & 16 & 22,9 \\
Ambos padres & & \\
Solo madre & 34 & 48,5 \\
Solo padre & 14 & 20,0 \\
Abuelos & 6 & 8,6 \\
Pensión - arriendo & 4 & 5,7 \\
Comparte pieza con amigos o familiares & 6 & 8,6 \\
Si & 6 & 8,6 \\
No & & \\
Asignaturas repetidas & 10 & 14,3 \\
Si & 60 & 85,7 \\
No & & \\
Realiza actividad deportiva & 10 & 14,3 \\
& 60 & 85,7 \\
\hline
\end{tabular}


media completa, correspondiendo el $37,1 \%$ a profesionales, técnicos y afines y el $48,5 \%$ dueñas de casa.

En los padres, el $40 \%$ presentó educa- ción básica completa y el 17,2\% enseñanza superior. De ellos, el 25,7\% correspondieron a profesionales, técnicos y afines y el $25,7 \%$ presentaban otros trabajos (Tabla 2).

Tabla 2. Perfil educacional de los padres de alumnos de Enfermería. Universidad de La Frontera, 2009.

\begin{tabular}{lcc}
\hline Variable & f & \% \\
\hline Años de estudio de la madre & 16 & 22,9 \\
Enseñanza Básica completa & 12 & 17,1 \\
Enseñanza Básica incompleta & 12 & 17,1 \\
Enseñanza Media completa & 20 & 28,6 \\
Enseñanza Media incompleta & 10 & 14,3 \\
Enseñanza Superior & & \\
Años de estudio del padre & 28 & 40 \\
Enseñanza Básica completa & 8 & 11,4 \\
Enseñanza Básica incompleta & 14 & 20 \\
Enseñanza Media completa & 8 & 11,4 \\
Enseñanza Media incompleta & 12 & 17,2 \\
Enseñanza Superior & & \\
Ocupación de la madre & 26 & 37,1 \\
Profesionales, técnicos y afines & 2 & 2,9 \\
Vendedores y afines & 2 & 2,9 \\
Artesanos y operarios & 6 & 8,6 \\
Trabajadores en servicios personales y afines & 34 & 48,5 \\
Dueña de casa & & \\
Orupación del padre & 18 & 25,7 \\
Geresionales, técnicos y afines & 6 & 8,5 \\
Empleados de oficina y afines & 4 & 5,7 \\
Vendedores y afines & 8 & 11,4 \\
Agricultores, ganaderos, pescadores y afines & 2 & 2,9 \\
Conductores de medios de transporte y afines & 10 & 14,3 \\
Obreros y jornaleros & 2 & 2,9 \\
Trabajadores en servicios personales y afines & 2 & 2,9 \\
Otros trabajos & 18 & 25,7 \\
\hline
\end{tabular}

${ }^{\star}$ Otros trabajos: Fuerzas Armadas y Carabineros; cesantes.

En un segundo nivel de análisis, se identificaron los valores centrales y secundarios expresados en los alumnos y su asociación con las variables sociodemográficas y curriculares de ellos.

En los valores centrales: la puntuación promedio global fue 4,8 , con una DS de 0,73 y rango de 3,6 a 6,7 .

Considerando la escala Likert de la en- cuesta de uno a siete, se identificó valores no considerados importantes por parte de los alumnos (valor menor a 3 puntos), entre ellos poder social y seguridad nacional. De los considerados importantes, los valores centrales con mayor puntuación fueron dar sentido a la vida, libertad, autorrespeto, seguridad familiar y trabajo (Tabla 3 ). 
Tabla 3. Valores centrales en alumnos de Enfermería. Universidad de La Frontera, año 2009.

\begin{tabular}{|c|c|c|c|c|c|c|c|}
\hline & \multicolumn{2}{|c|}{-1} & Promedio & DS & $\mathrm{P} 50$ & Mín. & Máx \\
\hline & $\mathrm{f}$ & $\%$ & 5,9 & 1,15 & 6 & 3 & 7 \\
\hline Armonía interna & - & - & 5,7 & 1,7 & 6 & 0 & 7 \\
\hline Poder social & 8 & 11,4 & 1,9 & 2,03 & 2 & -1 & 7 \\
\hline Placer & - & - & 4,1 & 1,56 & 4 & 2 & 7 \\
\hline Libertad & - & - & 6,1 & 1,08 & 6 & 2 & 7 \\
\hline Trabajo & - & - & 6,1 & 0,91 & 6 & 3 & 7 \\
\hline Vida Espiritual & - & - & 5,0 & 1,49 & 6 & 2 & 7 \\
\hline Sentimiento pertinencia & - & - & 5,0 & 1,35 & 5 & 3 & 7 \\
\hline Orden Social & - & - & 4,9 & 1,47 & 5 & 2 & 7 \\
\hline Vida excitante & - & - & 4,3 & 1,71 & 5 & 0 & 7 \\
\hline Dar sentido a la vida & - & - & 6,3 & 0,90 & 7 & 3 & 7 \\
\hline Buenos modales & - & - & 5,6 & 1,54 & 6 & 2 & 7 \\
\hline Riqueza & - & - & 3,0 & 1,41 & 3 & 0 & 7 \\
\hline Seguridad nacional & - & - & 2,8 & 1,72 & 3 & 0 & 6 \\
\hline Autorrespeto & - & - & 6,0 & 1,29 & 6 & 3 & 7 \\
\hline Reciprocidad de favores & - & - & 4,8 & 1,55 & 5 & 1 & 7 \\
\hline Creatividad & - & - & 4,8 & 1,36 & 5 & 2 & 7 \\
\hline Mundo en paz & - & - & 5,7 & 1,39 & 6 & 3 & 7 \\
\hline Respeto por tradición & 2 & 2,9 & 3,8 & 2,19 & 3 & -1 & 7 \\
\hline Amor maduro & - & - & 5,2 & 1,78 & 6 & 0 & 7 \\
\hline Autodisciplina & - & - & 4,9 & 1,79 & 6 & 0 & 7 \\
\hline Distanciamiento & - & - & 3,2 & 2,02 & 3 & 0 & 7 \\
\hline Seguridad familiar & - & - & 6,0 & 1,37 & 6 & 2 & 7 \\
\hline Reconocimiento Social & - & - & 4,5 & 1,59 & 5 & 1 & 7 \\
\hline Unión naturaleza & - & - & 4,0 & 1,62 & 4 & 0 & 6 \\
\hline Identidad nacional & - & - & 3,7 & 1,83 & 3 & 0 & 7 \\
\hline Vida variada & - & - & 4,5 & 1,66 & 5 & 0 & 7 \\
\hline Sabiduría & - & - & 5,4 & 1,36 & 6 & 2 & 7 \\
\hline Autoridad & - & - & 3,3 & 1,85 & 3 & 0 & 7 \\
\hline Amistad verdadera & - & - & 5,8 & 1,09 & 6 & 3 & 7 \\
\hline Mundo de belleza & - & - & 4,3 & 1,75 & 4 & 0 & 7 \\
\hline Justicia social & - & - & 5,6 & 1,45 & 6 & 2 & 7 \\
\hline Puntuación promedio tota & & & 4,8 & 0,73 & 4,8 & 3,6 & 6,7 \\
\hline
\end{tabular}

Datos expresados en frecuencias y porcentajes (\%) y medidas de resumen

-1 : Opuesto a mis valores

0,1 y 2 : Nada importante

3, 4 y 5: Importante

6: Muy importante

7: De suma importancia.

En los valores secundarios: la puntuación promedio global de las preguntas consideradas en esta dimensión fue de 5 puntos, puntaje por sobre la mediana, con una tendencia positiva, y una DS de 0,73 y rango de 3,6 a 6,7 .

Se identificó sólo un valor considerado no importante por parte de los alumnos (valor menor a 3 puntos), ser atrevido, entendido como aquel que busca aventuras y riesgos. De los considerados importantes, los valores centrales con mayor puntuación fueron lealtad, responsabilidad e inteligencia, con puntaje mayor o igual a 6 (Tabla 4 ). 
Tabla 4. Valores secundarios en alumnos de Enfermería. Universidad de La Frontera, 2009.

\begin{tabular}{|c|c|c|c|c|c|c|c|}
\hline & \multicolumn{2}{|c|}{-1} & \multirow{2}{*}{ Promedio } & \multirow{2}{*}{ DS } & \multirow{2}{*}{ P50 } & \multirow{2}{*}{ Mín. } & \multirow{2}{*}{ Máx. } \\
\hline & $\mathrm{f}$ & $\%$ & & & & & \\
\hline Independencia & - & - & 5,2 & 1,32 & 6 & 3 & 7 \\
\hline Moderado & - & - & 4,4 & 1,64 & 4 & 0 & 7 \\
\hline Leal & - & - & 6,3 & 0,74 & 6 & 5 & 7 \\
\hline Ambición & 2 & 2,9 & 3,6 & 2,03 & 3 & -1 & 7 \\
\hline Abierto & - & - & 5,5 & 1,43 & 6 & 2 & 7 \\
\hline Humilde & 2 & 2,9 & 4,8 & 1,92 & 5 & -1 & 7 \\
\hline Atrevido & 4 & 5,7 & 2,5 & 2,17 & 2 & -1 & 7 \\
\hline Protector medio ambiente & - & - & 4,4 & 1,87 & 5 & 0 & 7 \\
\hline Influyente & - & - & 3,5 & 1,79 & 4 & 0 & 7 \\
\hline Honrar a padres y mayores & - & - & 5,4 & 1,52 & 6 & 2 & 7 \\
\hline Elegir metas & - & - & 5,6 & 1,38 & 6 & 2 & 7 \\
\hline Sano & - & - & 5,9 & 1,15 & 6 & 2 & 7 \\
\hline Capaz & - & - & 5,9 & 0,96 & 6 & 4 & 7 \\
\hline Aceptación en la vida & 2 & 2,9 & 4,2 & 2,15 & 5 & -1 & 7 \\
\hline Honestidad & - & - & 5,9 & 1,27 & 6 & 2 & 7 \\
\hline Cuido imagen & - & - & 4,5 & 1,79 & 5 & 0 & 7 \\
\hline Obediente & - & - & 5,3 & 1,42 & 6 & 2 & 7 \\
\hline Inteligencia & - & - & 6,0 & 0,70 & 6 & 4 & 7 \\
\hline Ayuda a los demás & - & - & 5,5 & 1,43 & 6 & 1 & 7 \\
\hline Disfrutar la vida & 6 & 8,6 & 4,4 & 2,32 & 5 & -1 & 7 \\
\hline Devoción & 2 & 2,9 & 4,3 & 2,20 & 5 & -1 & 7 \\
\hline Responsabilidad & - & - & 6,1 & 0,83 & 6 & 4 & 7 \\
\hline Curiosidad & 2 & 2,9 & 4,7 & 1,88 & 5 & -1 & 7 \\
\hline No rencoroso & - & - & 4,9 & 1,76 & 5 & 0 & 7 \\
\hline Logro de éxitos & - & - & 5,3 & 1,29 & 6 & 3 & 7 \\
\hline Limpieza & - & - & 5,8 & 1,43 & 6 & 2 & 7 \\
\hline Autoindulgencia & 4 & 5,7 & 3,6 & 2,26 & 4 & -1 & 7 \\
\hline Puntuación promedio total & & & 5,0 & 0,73 & 5,0 & 3,6 & 6,7 \\
\hline
\end{tabular}

Datos expresados en frecuencias y porcentajes (\%) y medidas de resumen -1: Opuesto a mis valores

0,1 y 2 : Nada importante

3, 4 y 5: Importante

6: Muy importante

7: De suma importancia.

En relación a las asociaciones significativas hubo significancia entre Valores Centrales y nivel de avance curricular $(\mathrm{p}=0,026)$ (Tabla 5) y entre etnia mapuche con Valores
Secundarios $(\mathrm{p}<0,0001)$ (Tabla 6). No existió relación de significancia entre Valores Centrales según sexo y etnia (Tabla 5). 
Tabla 5. Valores Centrales según variables demográficas-curriculares en alumnos de Enfermería. Universidad de La Frontera, 2009.

\begin{tabular}{lcccc}
\hline & Promedio & DS & Frec. & p \\
\hline Hombres & 4,96 & 0,69 & 14 & \\
Mujeres & 4,71 & 0,73 & 56 & $0,2441^{\star}$ \\
\hline No repetido cursos & 4,68 & 0,65 & 60 & \\
Repetido cursos & 5,23 & 0,98 & 10 & $0,0266^{*}$ \\
\hline Etnia no mapuche & 4,71 & 0,77 & 58 & \\
Etnia mapuche & 4,98 & 0,45 & 12 & $0,1130 £$ \\
\hline
\end{tabular}

$\mathfrak{E}$ : ttest para varianzas distintas

*: ttest para varianzas iguales.

Tabla 6. Valores Secundarios, según variables demográficas curriculares en alumnos de Enfermería. Universidad de La Frontera, 2009.

\begin{tabular}{lcccc}
\hline & Promedio & DS & Frec. & p \\
\hline Hombres & 5,16 & 0,66 & 14 & \\
Mujeres & 4,90 & 0,74 & 56 & $0,2264^{*}$ \\
\hline No repetido cursos & 4,89 & 0,68 & 60 & \\
Repetido cursos & 5,32 & 0,94 & 10 & $0,0858^{*}$ \\
\hline Etnia no mapuche & 4,82 & 0,72 & 58 & \\
Etnia mapuche & 5,59 & 0,36 & 12 & $<0,0001 \mathfrak{}$ \\
\hline
\end{tabular}

$\mathfrak{E}$ : ttest para varianzas distintas

*: ttest para varianzas iguales.

\section{DISCUSIÓN Y COMENTARIO}

Los valores y ética en Enfermería constituyen un componente fundamental, en el momento de estudiar los comportamientos en la práctica profesional. Así, se tiene los principios que regulan conductas, motivaciones, cambios y transformaciones que se suceden a través del tiempo $(3,6)$. Los objetivos de la asistencia y docencia han de converger en el consenso de aquellos valores y referentes éticos más relevantes de la praxis, ajustados a los actuales paradigmas que orienten el trabajo disciplinar, en el ejercicio profesional (6).

La educación en Enfermería tiene la responsabilidad de fortalecer los valores per- sonales en el estudiantado, incorporando aspectos profesionales, motivando la defensa de sus intereses, para reconocer los valores de la realidad sociocultural en la cual están inmersos. En la medida que se logre confluir hacia esta realidad, se podrá advertir que existe "una jerarquía objetiva que se revela a quien comprende de alguna manera determinados valores" (14), diseñando un ambiente que favorezca el éxito de la vida personal y profesional.

En otro ámbito, el Código Deontológico de la Sociedad Cubana de enfermeras señala que se ha generado un aumento en el desinterés por la profesión, situación que conlleva a una disminución de la calidad de la atención brindada a los usuarios. En situaciones como 
ésta, el resquebrajamiento de los valores éticos profesionales, aun de los universalmente reconocidos, desempeñan una importante función (15).

Es por ello que algunos autores consideran oportuno incursionar en este tema y su relación en la formación de alumnos de Enfermería, con base en los valores personales, que pueden conformar el modelo del perfil humano que tipifique a la(el) enfermera(o) (16). Así, cuando los alumnos establecen relaciones interpersonales en su práctica clínica, esta situación debe ser trascendente, con los elementos necesarios para una adecuada interacción con las personas a su cuidado, más aún en la relación de ayuda, de lo contrario se puede ver obstaculizado su saber, saber ser, saber hacer y saber convivir, limitando asimismo el aprendizaje significativo que afecta el nivel de competencia dentro de las diferentes áreas de enfermería (17).

Entre las condiciones para el logro de una relación positiva, como se señaló anteriormente, deben existir los llamados elementos estimuladores tanto del crecimiento interpersonal como del ejercicio de actitudes básicas para la comunicación, entre ellos la congruencia, actitud positiva incondicional, respeto y empatía (18), los que de alguna u otra forma han sido manifestados por la población estudiada.

$\mathrm{Al}$ respecto, un estudio encontró que el tema central giraba en torno a las actitudes básicas con enfoque en la persona, donde los alumnos expresaron la importancia del proceso de autoconocimiento y de autoaceptación, como parte fundamental de la persona que brinda los cuidados de Enfermería (14). Situación que en su momento se relacionó con la Teoría de Rogers (19), que hace alusión a los seres humanos unitarios, mencionando que el hombre es un todo unificado que posee su propia integridad y manifiesta características que son más que diferentes a la suma de sus partes.

En relación a los hallazgos, éstos muestran una tendencia positiva en los valores centrales y se destaca como no importantes el poder social y seguridad nacional, situación que podría estar permeada por las etapas de la vida y consecuencia de procesos políticos sociales nacionales. Asimismo, se destacan como importantes: dar sentido a la vida, libertad, autorrespeto, seguridad familiar y trabajo, creencias que nos muestran una conducta aceptada socialmente, donde por la etapa de la vida en que se encuentran influyen las emociones, sentimientos y razonamiento. En los valores secundarios, al igual que los centrales muestran una tendencia positiva y se destacan como importantes: la lealtad, responsabilidad e inteligencia, valores altruistas, situación que llama la atención en un mundo marcado por la competitividad, eficiencia, eficacia y rentabilidad, con deterioro de las relaciones humanas, basados en el bienestar material individual y en el descuido de la dignidad de la persona. Por otra parte conocemos que los valores determinan las normas morales, es decir establecen un modelo de conducta aceptable en una sociedad, lo que, al destacar los valores señalados, muestra un prototipo de jóvenes con una relación valórica. Respecto de la significancia entre Valores Centrales y nivel de avance curricular, es explicable por el avance en edad, madurez y ciclo académico cursado.

Limitan la discusión el hecho de contar con limitadas publicaciones acerca del tema, situación que se corrobora en el trabajo de McNeese-Smith, publicado en Journals of Administración (20), donde refiere que muy poca investigación se ha realizado respecto de los valores y si éstos difieren según la edad, generaciones o fases del trabajo (clínico, administrativo, comunitario). Otros autores (21) aducen que los académicos de Enfermería tienen el desafío de enseñar valores básicos, que representan el cuidado profesional de la disciplina, no sólo con el propósito de integrar la educación basada en valores a través de todo el currículo sino que es materia esencial para asegurar que los estudiantes de Enfermería puedan aplicar los valores abs- 
tractos de la dignidad humana, la integridad, la autonomía, el altruismo y la justicia social en la práctica clínica. De esta forma, los valores de integración a través del currículo proporcionan los aspectos conceptuales, morales y prácticas de aprendizaje necesarias para garantizar en las enfermeras(os) el futuro del cuidar.

Finalmente, un estudio sobre la socialización de la profesión de enfermería encontró que la exposición a la educación influye positivamente en la capacidad de pensamiento crítico y en el fortalecimiento de los valores profesionales, por lo tanto la docencia debe asumir que es superior en estas características, en este estudio los profesores valoran el logro más alto $(\mathrm{p}=0,001)$, y los estudiantes adjudican mayor valor al cumplimiento de la meta y la benevolencia $(\mathrm{p}=0,001)$. Aspectos similares se encontraron en el ámbito del logro de metas, como valor principal manifestado por los estudiantes, alcanzando prácticamente el 50\% de las preferencias, puntuando alrededor de 5,3 puntos (22).

A la postre, podemos decir que el estudio de los valores ha cobrado especial trascendencia, debido a la complejidad de un mundo caracterizado por el relativismo moral y crisis de valores, lo que repercute en cambios constantes y profundos, especialmente en las costumbres y sistema de valores. En este contexto se encuentran insertos los alumnos encargados del cuidado de la salud, situación que podría ser gravitante en futuros profesionales de la salud, quienes son garantes de los derechos y deberes de los usuarios o pacientes y podrían estar expuestos a lo que se ha llamado "vulnerabilidad moral", explicada por la diversidad de condiciones de vida, faltas de equidad no resueltas en el acceso a bienes y servicios, y diferente práctica jurídica. Esto significa un riesgo a tomar en cuenta en los planes de enseñanza y educación pública (23).

\section{REFERENCIAS}

1. Barylko J. Los valores y las virtudes. Buenos Aires: Emecé; 2000.

2. Maslow A. Motivation and personality. New York: Harper and Row Publishers; 1954.

3. Rokeach M. The nature of human values. New York: Free Press; 1973.

4. Jonas H. Técnica, medicina y ética. Barcelona: Paidós Básica; 1997. p. 297.

5. Lolas F. Bioética y antropología médica. Santiago, Chile: Mediterráneo; 2000. p. 23.

6. Falcó A, Tomás-Sábado J. La formación en valores profesionales de los estudiantes de enfermería. Síntesi bioètica i infermeria. 2004; 2(5): 1-2.

7. Weis D, Schank M. An instrument to measure professional nursing values. J Nurs Scholarsh [Internet]. 2004[citado 25 abril 2010]; 32(2): 201-04. Disponible e $n:$ t t p : / / w w w 3 . interscience.wiley.com/journal/119052275/ abstract?CRETRY=1\&SRETRY

8. Scheler M. El formalismo en la ética y la ética material de los valores. Tomo I. Buenos Aires: Revista de Occidente. 1948. p. 42.

9. Ortega y Gasset J. El tema de nuestro tiempo. O.C. III. 5a ed. Madrid: Revista de Occidente.1962. p 35.

10. Arratia A. Aspectos éticos vulnerados en situaciones de violencia: su importancia en la formación de Enfermería. Invest. educ. enferm. [Internet]. 2005 [citado 25 abril 2010]; 23(2):104-16. Disponible en: www.scielo.org.co/pdf/iei/iee/v23n2/ v23n2a09.pdf.

11. Villalapos G, López A. El libro de los valores. 6a ed. Barcelona: Planeta; 1998.84p.

12. López A. El conocimiento de los valores. Navarra: Verbo Divino; 1989. 35p. 
13. Schwartz S, Bilsky W. Toward a Psychological Structure of Human Values. Journal of Personality and Social Psychology. 1987; 53: 550-62.

14. Spaemann R. Ética: Cuestiones fundamentales. 4a ed. España: EUNSA, 1995. $88 \mathrm{p}$.

15. Código Deontológico de la Sociedad $\mathrm{Cu}-$ bana de Enfermeras para la profesión de Enfermería en la República de Cuba. Fundamentación. La Habana. 2001. p. 2.

16. Fabelo R. Los valores y los desafíos actuales. México: BUAP, 2001.

17. Hernández G, Cabrera C, Garay F. El desarrollo humano en la formación profesional de enfermería. Rev Enferm IMSS [Internet].2004 [citado 10 abril 2010]; 12(1): 3-7. Disponible en: http://www.imss.gob. $\mathrm{mx} / \mathrm{NR} /$ rdonlyres/90DB7A8D-1F584321-B084CAC919B5C3D2/0/1_37.pdf

18. Rogers C. Psychothérapie et relations humaines [Internet]. Madrid; Barcelona: Alfaguara; 1971 [citado 18 mayo 2010]. 284-365p. Disponible en: http://roble. unizar.es/

19. Leddy S. Bases conceptuales de la enfermería profesional. En: Hernández G, Cabrera C, Garay F. El desarrollo humano en la formación profesional de enfermería. México, Juárez: Rev Enferm IMSS;
2004. 12(1): 3-7.

20. Mc Neese - Smith DK. Nursing Values and a Changing Nurse Workforce: Values, Age and Job Stages. J Nursing Adm [Internet]. 2003 [citado18 mayo 2010]; 33(5): 26070. Disponible en: http://journals.lww. com/jonajournal/Abstract/2003/05000/ nursing_Values_and_a_Changing_Nurse_Workforce_.2.aspx

21. Fahrenwald N, Bassett S, Tschetter L, Carson P, White L, Winterboer V. Teaching Core Nursing Values. J Prof Nurs [Internet]. 2005 [citado 18 mayo 2010]; 21(1): 46-51. Disponible en: http://www. sciencedirect.com/

22. Saarman L, Freitas L, Rapps J, Riegel B. The relationship of education to critical thinking ability and values among nurses: Socialization into professional nursing. J Prof Nurs [Internet].1992 [citado18 mayo 2010]; 8(1): 26-34. Disponible en: http://www.ncbi.nlm.nih.gov/ pubmed/1573111

23. Lolas F. La bioética en el contexto de los programas globales de salud. Rev. Panam. Salud Pública [Internet]. 1999 [citado 18 agosto 2011]; 6(1): 65-8. Disponible en: http://dx.doi.org/10.1590/S102049891999000600017. 